\title{
Efficient Utilization of FlexRay Network Using Parameter Optimization Method
}

\author{
Y. X. Wang, Y. H. Xu, and Y. N. Xu
}

\begin{abstract}
FlexRay is a high rate of bus communication standard in-vehicle network system, which takes time division multiple access (TDMA) and flexible time division multiple access (FTDMA) media mechanism to transmit messages flexibly, efficiently and reliably. However, due to protocol constraints and multiple design objectives, it is not an easy task to design a FlexRay schedule. In this paper, to achieve more efficient use of FlexRay network, an algorithm to obtain the optimal length of static messages is proposed, and the messages which are longer than the optimal length are assigned to dynamic segment. Then, according to the important level of dynamic messages, a frame ID allocation scheme by using optimal scheduling algorithm of dynamic programming and multi-stage decision for dynamic messages is proposed. After analyzing the worst delay time of the static and dynamic frames, the dynamic segment length optimization algorithm which aimed at minimizing the worst delay time of messages is put forward. Finally, according to the results which are the application of the algorithms to the FlexRay-based vehicle chassis control system, the static slot utilization rate reached $86.5 \%$, the length of dynamic segment which transmits aperiodic messages is reduced by $\mathbf{4 3 . 4} \%$.
\end{abstract}

Index Terms-FlexRay, frame ID allocation, network utilization, parameter optimization, worst delay time.

\section{INTRODUCTION}

FlexRay is a new standard of network communication system which provides a high speed serial communication, fault tolerant communication between electronic devices for future automotive applications. FlexRay supports a time-triggered scheme and an optional event-triggered scheme. The upper bound of the data rate is $10 \mathrm{Mbps}$ and it provides two channels for redundancy.

Each communication cycle contains the static segment (ST), dynamic segment (DYN), symbol window (SW), and network idle time (NIT). The static segment and dynamic segment are used for transmitting messages. The payload length shall be fixed and identical for all frames sent in the static segment of a communication cycle, for these frames the payload length field shall be transmitted with the payload length of static slot. However, the payload length may be different for different frames in the dynamic segment. A scheduling algorithm that long static messages are allocated to the dynamic segment is presented [1], [2], whereas there is no specific frame ID allocation for the periodic messages allocated to the dynamic segment. In [3], an algorithm named

Manuscript received September 23, 2015; revised November 12, 2015 This work was supported by the National Science Foundation of China (61361003).

The authors are with the division of electronic and communication engineering of Yanbian University, Yanji, China (e-mail: 2014050258@ybu.edu.cn,xuyh@ybu.edu.cn, ynxu@ybu.edu.cn). recursive qualification which can prevent the propagation delay to the next cycle and reduce the absolute delay time is proposed, whereas the study did not consider the variable of parameter pLatesTx. In [4], under considering protocol overhead and unused network resources, an algorithm that can get the optimal static slot length is put forward, and longer messages are divided into multi-messages to be transmitted in the static segment. This improves the efficient utilization of network, while the communication cycle is prolonged because of the increase of the protocol overhead. An algorithm providing a tight upper bound to the worst case response times to assign identifiers to frames is studied in [5]. The stability region for the random arrival of messages and the timing analysis in dynamic segment are studied in [6], [7].

In this paper, aiming at the problem of efficient FlexRay network utilization and fast messages transmission, a static slot length optimization algorithm, a frame ID optimization algorithm and a dynamic segment length optimization algorithm are proposed. Messages are divided into two kinds that are periodic and aperiodic based on the type of message, most periodic messages are transmitted on the static segment, longer periodic messages and aperiodic messages are transmitted on the dynamic segment. To get the number of periodic messages as much as possible, the optimal length of the static slot is determined by the constraint of network utilization. Then, based on the important level of dynamic messages, the frame ID allocation scheme by using optimal scheduling algorithm of dynamic programming and multi-stage decision for dynamic messages is proposed. After analyzing the worst delay time of the static and dynamic frames, the algorithm which aiming at minimizing the worst delay time of messages to find the optimal length of the dynamic segment is put forward.

In section II, FlexRay frame format is briefly reviewed. Section III presents the static slot length optimization algorithm, frame ID optimization algorithm and dynamic segment length optimization algorithm. Section IV presents the simulation results of proposed algorithms to vehicle chassis control system.

\section{FEXRAY FRAME Format}

FlexRay frame consists of the header segment, payload segment, and trailer segment [8]-[10]. The frame ID defines the slot in which the frame should be transmitted. If missed the corresponding slot of the communication cycle, the static message would be transmitted in the next communication cycle, whereas the dynamic message might have to wait for one or more communication cycles. So the length of dynamic segment and the frame IDs should be selected properly to reduce the impact of the delay caused by the uncertainty of 
messages. All frames transmitted on FlexRay network encoded by format of Fig. 1.

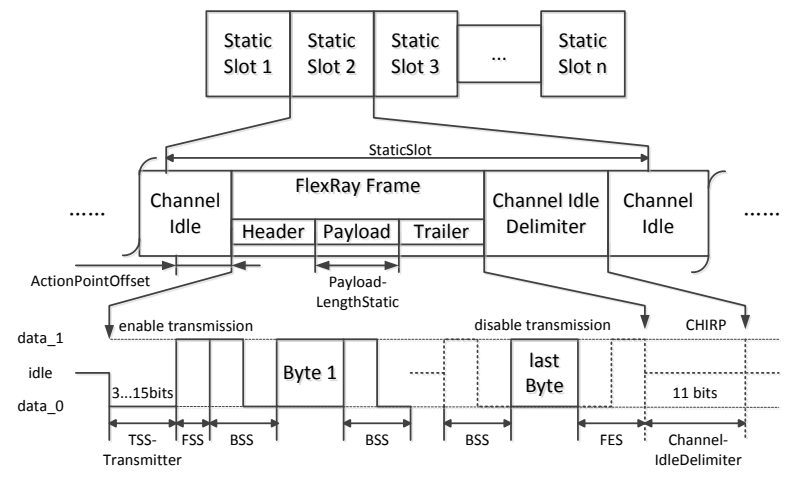

Fig. 1. Frame encoding in the static segment.

In Fig. 1, the Transmission Start Sequence (TSS) is 3 15 bits, Frame Start Sequence (FSS) is 2 bits, Byte Start Sequence (BSS) is 2 bits, Frame End Sequence (FES) is 2 bits, DYN Trailing Sequence (DTS) is 2 bits, added after the FES in the case of dynamic segment. Every byte has a BSS. When a $n$ byte message is to be transmitted, the length of static frame and dynamic frame are given by

$$
\begin{gathered}
L_{S F r}=T S S+F S S+80+n(8+2)+F E S \\
L_{D F r}=T S S+F S S+80+n(8+2)+F E S+D T S
\end{gathered}
$$

where, $L_{S F r}$ and $L_{D F r}$ are the sizes of static frame and dynamic frame respectively, 8 bytes is the summation of header and trailer segment of a frame.

Hence, the length of static slot can be calculated by

$$
\begin{aligned}
& L_{S T}=\operatorname{ceil}\left[\left(\left(L_{S F r}+C I D\right) \times t_{B i t M a x}+\operatorname{MinPD}+\operatorname{Max} P D\right)\right. \\
& \left.\times\left(t_{M T} \times(1-C D M)\right)^{-1}\right]+2 \times A P O
\end{aligned}
$$

where, $L_{S T}$ is the length of static slot. Ceil is the top integral function, whose value is the smallest integer greater than the independent variable or equal to it. The Action Point Offset (APO) is 1-63 MT, Channel Idle Delimiter (CID) is 11 bits, $t_{\text {BitMax }}$ is the maximum bit time taking into account the allowable clock deviation of each node, Min Propagation Delay (MinPD) is $0-2.5 \mu \mathrm{s}$, Max Propagation Delay (MaxPD) is $0-2.5 \mu \mathrm{s}, t_{M T}$ is the duration of the cluster wide nominal macrotick expressed in $\mu$ s. Clock Deviation Max (CDM) is 0.0015 .

When one frame is transmitted in the dynamic segment, the number of minislots (MS) can be calculated by

$$
L_{D Y N}=1+\operatorname{ceil}\left(\frac{t_{\text {BitMax }}\left(L_{D F r}+D T S L o w\right)}{t_{M T}(1-C D M) t_{M S}}\right)+D S I P
$$

where, DTSLow is 1 bit, $t_{M S}$ is the duration of a minislot, DSIP is dynamic slot idle phase.

\section{PARAMETER OPTIMIZATION AlgORITHM}

When a set of messages needed to be transmitted on
FlexRay network are given, the messages are divided into two kinds. This paper has proposed three optimization algorithms to determine FlexRay network core parameters like the length of static slot, the frame ID allocation, and the length of dynamic segment. Assume that the number of message type is two and all messages are transmitted on one channel. The design is based on the parameters shown in Table I. Where, $S S N$ is the number of static slots.

\begin{tabular}{l|l|l|l}
\multicolumn{4}{|c}{ TABLE I: NETWORK PARAMETERS } \\
\hline \hline Element & Length & Element & Length \\
\hline $\mathrm{MT}$ & $1 \mu \mathrm{s}$ & MinPD & $1 \mu \mathrm{s}$ \\
\hline $\mathrm{MS}$ & $3 \mathrm{MT}$ & MaxPD & $2 \mu \mathrm{s}$ \\
\hline $\mathrm{t}_{\text {BitMax }}$ & $0.1 \mu \mathrm{s}$ & $\mathrm{SSN}$ & 27 \\
\hline Bit Rate & $10 \mathrm{Mbps}$ & $\mathrm{TSS}$ & $10 \mathrm{bit}$ \\
\hline $\mathrm{DSIP}$ & $1 \mathrm{MS}$ & APO & $1 \mathrm{MT}$ \\
\hline NIT & $230 \mathrm{MT}$ & $\mathrm{SW}$ & $10 \mathrm{MT}$ \\
\hline \hline
\end{tabular}

\section{A. Static Slot Length Optimization Algorithm}

The length of static slot is determined by the longest message, when the number of long messages is few, the network utilization will be low for the unused network resource. However, if longer messages are assigned to the dynamic segment, not only the network utilization is increased, but also the communication cycle is decreased by the reduction of the protocol overhead.

When $S$ periodic messages are given, they are $S T_{i}(i=1,2 \cdots S)$, the best length of message is a variable $x$ (byte), using (1) and (3), the relationship between static slot length and $x$ is

$$
L_{S T}(x)=2+\operatorname{ceil}\left(\frac{13.5+x}{0.9985}\right)
$$

A set $A_{x}$ contains the messages whose length is less than or equal to $x$, the number of these messages is $n_{x}$, the static slots utilization is defined as follows :

$$
U_{S T}(x)=\left(n_{x} L_{S T}(x)\right)^{-1} \sum_{n=1}^{n_{x}} L_{S T}\left(l_{n}\right)
$$

where, $l_{n}$ is the length of message in set $A_{x}$.

When $x$ is small, the static slots utilization will be high, while the burden of dynamic segment will increase because the number of messages transmitted on static segment is decreased. Thus, it is better that more messages are transmitted on static segment. The ratio of messages transmitted on static segment of the total periodic messages can be expressed as:

$$
P_{S T}(x)=\frac{n_{x}}{S}
$$

With the increase of $x$, the static slots utilization will decrease, however, the ratio (7) will increase. Therefore, the best length of message can be got through the summation of $U_{S T}$ and $P_{S T}$.

$$
S T(x)=U_{S T}(x)+P_{S T}(x),\left(\operatorname{Min}_{S T} \leq x \leq \operatorname{Max}_{S T}\right)
$$


where $\operatorname{Min}_{S T}$ is the length of the shortest message, $\operatorname{Max}_{S T}$ is the length of the longest message.

\section{B. Frame ID Optimization Algorithm}

In the dynamic segment, messages are transmitted based on frame IDs, a lower frame ID of the message will get higher priority than a higher frame ID. According to the different importance, aperiodic messages are assigned with important levels, different messages may have the same important level. Under the limitation of fixed dynamic segment length, messages are selected by optimal scheduling algorithm of dynamic programming to let the summation of important level be the maximum. Still follow the dynamic programming algorithm, the rest messages will be assigned to next cycle. Through multi-stage decision, all aperiodic messages will be assigned with an unique frame ID which is determined by the order of the screening results.

When $n$ messages are given, the length in terms of minislots of message $i(1 \leq i \leq n)$ is $l_{i}$, and the important level is $v_{i}$. The length of the dynamic segment is $d$. For each message only two options that selected or not can be chosen. So the problem can be described as: seeking out a vector $\left(x_{1}, x_{2}, \cdots x_{n}\right), x_{i} \in\{0,1\}, 1 \leq i \leq n$ to let $\sum_{i=1}^{n} l_{i} x_{i} \leq d \quad$ and $\sum_{i=1}^{n} v_{i} x_{i}$ be the maximum.

The optimal value of sub-problem of the problem is $m(i, j)$.

$$
\begin{gathered}
\max \sum_{k=i}^{n} v_{k} x_{k} \\
\sum_{k=i}^{n} l_{k} x_{k} \leq j, \quad x_{k} \in\{0,1\}, i \leq k \leq n
\end{gathered}
$$

where $m(i, j)$ is the optimal solution of sub-problem that the length of dynamic segment is $j$ and the optional messages are $i, i+1, \cdots, n$.

The recursive calculation equation of $m(i, j)$ can be established as:

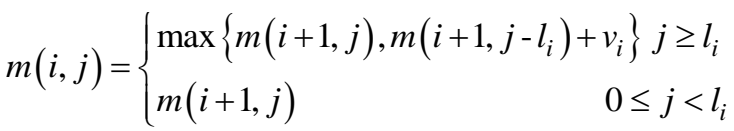

$$
\begin{aligned}
& m(n, j)=\left\{\begin{array}{lc}
v_{n} & j \geq l_{n} \\
0 & 0 \leq j<l_{n}
\end{array}\right.
\end{aligned}
$$

\section{Dynamic Segment Length Optimization Algorithm}

Two kinds of messages need to be transmitted on the dynamic segment: one is longer periodic messages assigned from the static segment, the other is aperiodic messages. The delay model of messages is shown in Fig. 2.

Periodic messages are assigned to the first few slots of the dynamic segment with higher priority (as $S_{4}$ in Fig. 2). The rest IDs are assigned to aperiodic messages.

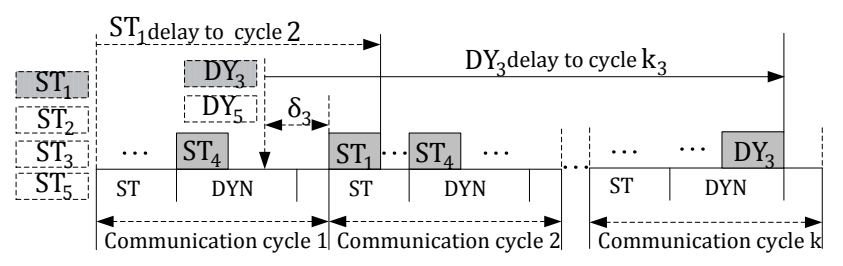

Fig. 2. Delay model of messages.

$$
D(M S)=d_{s t}+d_{d y} \quad\left(d_{\min } \leq d_{d y} \leq d_{\max }\right)
$$

where $D$ is the length of dynamic segment, $d_{s t}$ is the summation length in terms of minislots of periodic messages. $d_{d y}$ is the length in terms of minislots used for transmintting the aperiodic messages. $d_{\min }$ is the length needed by the longest aperiodic message transmitting on the last slot of the dynamic segment. $d_{\max }$ is the summation length of all aperiodic messages.

If the dynamic segment length is short, the worst delay time will be too large for many aperiodic messages are delayed to next cycle. In Fig. 2, a node send $g_{S T}$ messages at one time, the worst delay time due to the competition in one node for each message is $\left(g_{S T}-1\right)$ communication cycles. In the case of static message fail to be sent in the first communication cycle, it will be sent in the next cycle. So the worst delay time for each static message is described by

$$
W_{S T_{i}}=g_{S T_{i}} c_{c y c l e}+l_{S T_{i}}
$$

where $W_{S T_{i}}$ is the worst delay time of static message $S T_{i}$, $c_{\text {cycle }}$ is the communication cycle, $l_{S T_{i}}$ is the length of static message $S T_{i}$.

Similarly, the worst delay time for the competition in one node for an aperiodic message is $\left(g_{D Y}-1\right)$ communication cycles and $g_{D Y}$ is the number of aperiodic messages in one node. If an aperiodic message failed to transmit in the first cycle, it would wait for one or more cycles due to the random variation of aperiodic messages, such as $\mathrm{DY}_{3}$ is transmitted in cycle $k_{3}$ in Fig. 2. The aperiodic messages ready to be transmitted whose IDs are less than $D Y_{i}$ 's are placed in set $B_{i}$ in cycle $k_{i}$, set the number of set $B_{i}$ is $n_{k_{i}}$, the length of each element is $l_{D Y_{J}}\left(1 \leq j \leq n_{k_{i}}\right)$. The worst delay time of aperiodic message is

$$
W_{D Y_{i}}=\delta_{i}+c_{c y c l e}\left(g_{D Y_{i}}+k_{i}-3\right)+\sum_{j=1}^{n_{k_{i}}} l_{D Y_{j}}+l_{D Y_{i}}
$$

where $W_{D Y_{i}}$ is the worst delay time of $D Y_{i}, \delta_{i}$ is the delay from request time to the end of the first communication cycle, $l_{D Y_{i}}$ is the size of message $D Y_{i}$. 
The optimum value of $d_{d y}$ can be obtained through the summation of (14) and (15).

$$
W=\sum_{i=1}^{n_{d}} W_{D Y_{i}}+\sum_{i=1}^{n_{s}} W_{S T_{i}}
$$

where $W$ is the worst delay time for all messages, $n_{s}$ is the number of the periodic messages, $n_{d}$ is the number of the aperiodic messages.

\section{Simulation Results}

In this paper, we focused on the FlexRay-based vehicle chassis control system. It consists of thirty-nine nodes, as noted in Table II and Table III.

TABLE II: EXAMPLE OF PERIODIC MESSAGE

\begin{tabular}{|c|c|c|c|}
\hline Message & Length & Message & Length \\
\hline ABS1 & 5 & DCT5 & 7 \\
\hline ABS2 & 5 & DCT6 & 4 \\
\hline ABS3 & 7 & SRS1 & 14 \\
\hline ESC1 & 2 & EPS1 & 4 \\
\hline ESC4 & 4 & ECM1 & 3 \\
\hline ESC5 & 6 & ECM2 & 12 \\
\hline ESC6 & 8 & ECM3 & 7 \\
\hline GW1 & 3 & ECM4 & 18 \\
\hline GW2 & 1 & ECM5 & 8 \\
\hline $\mathrm{ACC} 1$ & 4 & ECM6 & 8 \\
\hline DCT3 & 10 & DCT4 & 6 \\
\hline ACC2 & 6 & IP2 & 4 \\
\hline BCM1 & 1 & ECM7 & 12 \\
\hline BCM3 & 2 & ECM8 & 6 \\
\hline GW3 & 2 & PEPS3 & 6 \\
\hline SRS3 & 4 & DCT1 & 6 \\
\hline \multicolumn{4}{|c|}{ TABLE III: EXAMPLE OF APERIODIC MESSAGE } \\
\hline Message & \multicolumn{2}{|c|}{ Length } & $\begin{array}{c}\text { Important } \\
\text { Lavel }\end{array}$ \\
\hline ESC2 & \multicolumn{2}{|c|}{12} & 1 \\
\hline ACC3 & \multicolumn{2}{|c|}{16} & 5 \\
\hline BCM6 & \multicolumn{2}{|c|}{16} & 3 \\
\hline ЕCM9 & \multicolumn{2}{|c|}{28} & 6 \\
\hline PEPS1 & \multicolumn{2}{|c|}{18} & 4 \\
\hline SRS2 & \multicolumn{2}{|c|}{22} & 4 \\
\hline DCT2 & \multicolumn{2}{|c|}{16} & 2 \\
\hline
\end{tabular}

The chassis control system consists of eleven kinds of blocks: Anti-locked Braking System (ABS), Electronic Stability Controller (ESC), Gateway (GW), Adaptive Cruise Control (ACC), Body Control Module (BCM), Instrument Panel (IP), Passive Entry Passive Start (PEPS), Dual Clutch Transmission (DCT), Supplemental Inflatable Restraint System (SRS), Electric Power Steering system (EPS), and
Engine Control Module (ECM). The important level is altering from 1 to 6 . The lengths of shortest and longest periodic messages are 1 byte and 18 bytes respectively, the longest aperiodic message is ECM9. Using (14), $d_{\text {min }}=21 M S$ and $d_{\max }=83 M S$ can be got.

The static segment utilization rate is shown in Fig. 3. Where $U(x),(1 \leq x \leq 18)$ is the static slot utilization rate, and $P(x),(1 \leq x \leq 18)$ is the ratio of messages transmitted on the static segment of the total periodic messages. When $x$ changes from 1 to $18, U(x)$ gradually decreases from 1 , meanwhile $P(x)$ increases until 1. The optimal length of static slot is 24 MT when $x=8$ byte and $U(x)+P(x)$ is the maximum. The 5 messages which are longer than 8 bytes are DCT3, SRS1, ECM2, ECM4, and ECM7. Their frame IDs are from 1 to 5 , and $d_{s t}=51 M S$ can be got by using (4).

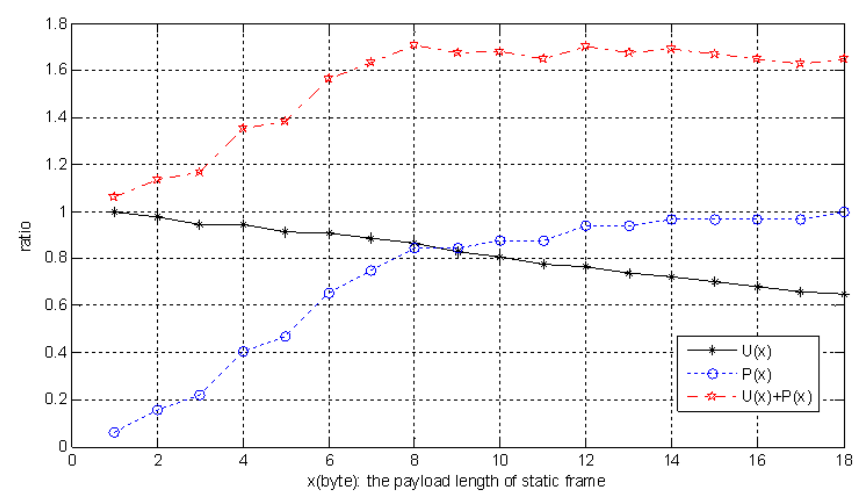

Fig. 3. Static slots utilization rate.

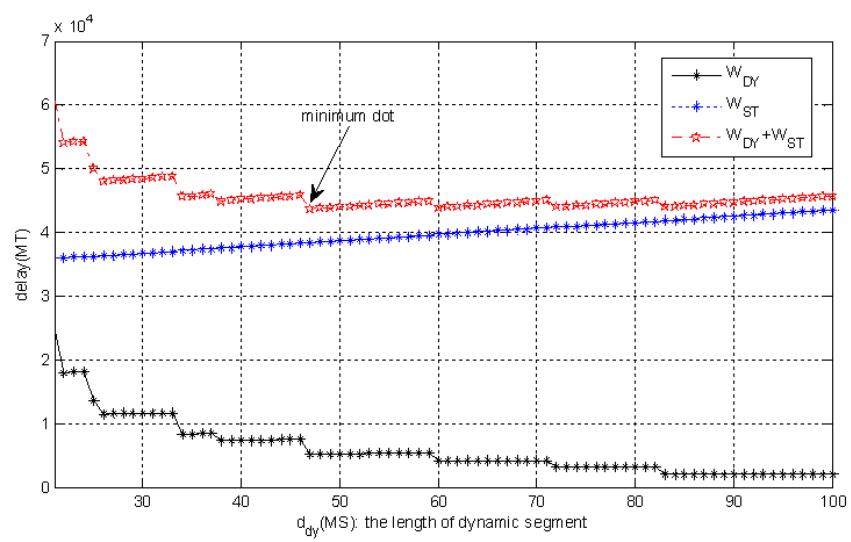

Fig. 4. Worst delay time of messages.

The worst delay time of messages is shown in Fig. 4. Where $W_{S T}$ and $W_{D Y}$ are the worst delay time of the periodic messages and the aperiodic messages respectively. When $d_{d y}$ alters from 83 to 100 , the value of $W_{D Y}$ is constant for all aperiodic messages can be transmitted in one communication cycle. The best value of $d_{d y}$ is $47 \mathrm{MS}$ when $W_{D Y}+W_{S T}$ is the minimum, so $D$ is equal to $98 \mathrm{MS}$. According to the frame ID optimization algorithm, the frame ID assignment for the aperiodic messages: the ACC3 frame ID is 6 , SRS2 frame ID is 7, PEPS1 frame ID is 8, BCM6 frame ID is 9, ECM9 frame ID is 10, DCT2 frame ID is 11, and ESC2 frame ID is 12.

The duration of communication cycle is 
$c_{\text {cycle }}=27 L_{S T}(8)+D+N I T+S W=1182 M T$. Finally, the static slot utilization rate reached $86.5 \%$. The FlexRay network utilization is defined from the static slot or the minislot which are used for message transmission in communication cycle [2], so network utilization with using the proposed algorithms is $61.5 \%$ which is much lower than that of $83.5 \%$ without using algorithm. Furthermore, the size of $d_{d y}$ is decreased from 83 MS to 47 MS by applying the algorithm, that is the size of the dynamic segment which used for transmitting the aperiodic messages is reduced by $43.4 \%$. Finally, the utilization of static segment and dynamic segment is further improved.

\section{CONCLUSION}

This paper carries out the optimization study of FlexRay network parameters. In the first step, the static slot length optimization algorithm is proposed by the constraint of the network utilization and the number of periodic messages which are transmitted on the static segment. In the second step, according to the important level of dynamic message, the frame ID allocation scheme by using optimal scheduling algorithm of dynamic programming and multi-stage decision for dynamic messages is proposed, besides, the flexibility of this algorithm is improved by the variation of important level. In the third step, after analyzing the worst delay time of the static and dynamic frames, the algorithm to find the optimal length of dynamic segment is put forward. Finally, according to the application of the algorithms to vehicle chassis control system, the static slot utilization rate reaches $86.5 \%$, the length of dynamic segment which transmits aperiodic messages is reduced by $43.4 \%$, and the network utilization rate is reduced to $61.5 \%$.

\section{REFERENCES}

[1] K. Park, M. Kang, and B. Kim. "A scheduling algorithm for reducing flexray message response time using empty minislots in dynamic segment," in Proc. the International Conference on Consumer Electronics (ICCE 2010), Digest of Technical Papers, 2010, pp. 307-308

[2] S. Byungseok and D. Lee, "Determining the length of static message for efficient use of FlexRay network," in Proc. the Conference 2010 SICE Anпиаl , 2010, pp. 563-566.

[3] J. K. Ho et al., "Priority-based scheduling of dynamic segment in FlexRay network," in Proc. International Conference on IEEE, Control, Automation and Systems, 2008, pp. 1036-1041.

[4] P. Inseok and M. Sunwoo, "FlexRay network parameter optimization method for automotive applications," Industrial Electronics IEEE Transactions, vol. 58, pp. 1449-1459, 2011.
[5] Z. Haibo, A. Ghosal, and M. D. Natale. "Timing analysis and optimization of flexray dynamic segment," in Proc. International Conference on IEEE Computer and Information Technology, pp. 1932-1939, 2010.

[6] B. Tanasa, U. Bordoloi, S. Kosuch, P. Eles, and Z. Peng, "Schedulability analysis for the dynamic segment of flexray: A generalization to Slot multiplexing," Real-Time and Embedded Technology and Applications Symposium, pp. 185-194, 2012.

[7] S. P. R. Kumar and L. J. Enkins, "Stability analysis of aperiodic messages scheduled in the dynamic segment of flexray protocol," in Proc. 2012 Third International Conference on Computing Communication and Networking Technologies, 2012, pp. 1-7.

[8] Y. N. Xu, Y. E. Kim, K. J. Cho, J. G. Chung, and M. S. Lim. "Implementation of Flexray protocol with an automotive Application," in Proc. IEEE International SoC Design Conference, 2008, vol. 02, pp. 25-28.

[9] Y. N. Xu, Y. E. Kim, K. J. Cho, J. G. Chung, and X. Huang, "Implementation of flexray CC and BG protocols with application to a robot system," Intl. Journal of Computer Aided Engineering and Technology, vol. 2, no. 1, pp. 112-122, 2010.

[10] FlexRay Consortium. FlexRay Communications System Protocol Specification v2.1 Revision A, 2005.

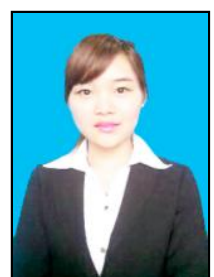

Y. X. Wang was born at Jilin province of China. She completed bachelor degree in the field of electronics and telecommunication, Yanbian University, Yanji, China, in 2013.

She is still pursuing her master degree in the School of Yanbian University, Yanji, China.

Her research interests include the in-vehicle network and automobile electronic control.

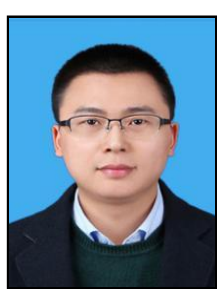

Y. H. Xu was born at Jilin province of China. He received the Ph.D. degree in electronics engineering from the Chonbuk National University, Korea, in 2014.

$\mathrm{He}$ is a lecturer of the division of electronic and communication engineering of Yanbian University, Yanji, China. His research interests include the automobile electronic control and network.

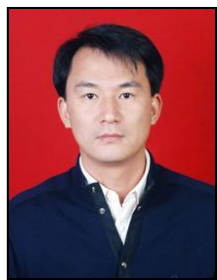

Y. N. Xu was born at Jilin province of China. He received the Ph.D. degree in electronics engineering from the Chonbuk National University, Korea, in 2009.

$\mathrm{He}$ is an associate professor of the division of electronics and communication engineering of Yanbian University, Yanji, China. His research interests include the in-vehicle network and automobile electronic control. 